\title{
Self-Consistent Coupling of DSMC Method and SOLPS Code for Modeling Tokamak Particle Exhaust
}

\author{
F. Bonelli ${ }^{1}$, S. Varoutis ${ }^{1}$, D. Coster ${ }^{2}$, Chr. Day $^{1}$, R. Zanino ${ }^{3}$ and JET Contributors \\ ${ }^{1}$ Karlsruhe Institute of Technology (KIT), Institute of Technical Physics, Vacuum \\ Department, Karlsruhe, Germany \\ ${ }^{2}$ Max-Planck-Institute for Plasma Physics (IPP), Garching, Germany \\ ${ }^{3}$ NEMO group, Dipartimento Energia, Politecnico di Torino, Torino, Italy \\ *EUROfusion Consortium, JET, Culham Science Centre, Abingdon, OX143DB, UK
}

Email contact of main author: stylianos.varoutis@kit.edu

\begin{abstract}
In this work, the investigation of the neutral gas flow in the JET sub-divertor area is presented, with respect to the interaction between the plasma side and the pumping side. The edge plasma side is simulated with the SOLPS code, while the sub-divertor area is modeled by means of the Direct Simulation Monte Carlo (DSMC) method, which in the last few years has been proved able to well describe rarefied, collisional flows in tokamak sub-divertor structures. Four different plasma scenarios have been selected and for each of them a userdefined, iterative procedure between SOLPS and DSMC has been established, using the neutral flux as the key communication term between the two codes. The goal is to understand and quantify in a self-consistent manner the mutual influence between the two regions, namely, how the particle exhaust pumping system controls the upstream plasma conditions. Parametric studies of the flow conditions in the sub-divertor, including additional flow outlets and variations of the cryopump capture coefficient have been performed as well, in order to understand their overall impact on the flow field. The DSMC analyses resulted in the calculation of both the macroscopic quantities, i.e. temperature, number density and pressure, and the recirculation fluxes towards the plasma chamber. The consistent values for the recirculation rates were found to be smaller than according to the initial standard assumption made by SOLPS.
\end{abstract}

\section{Introduction}

In this paper, the neutral deuterium gas flow behavior through the JET divertor is investigated by coupling two well-established numerical approaches, namely the SOLPS code package [1] and the Direct Simulation Monte Carlo (DSMC) Method [2].

In the last few years, significant effort [3-6] has been devoted to properly model the neutral gas flows in the divertor and sub-divertor areas in tokamak fusion reactors, for the neutral dynamics heavily influence the exhaust pumping process and the overall pumping efficiency, and therefore has directly influenced the design of the machine [7]. Nevertheless, from both the physics and the engineering point of view, the description of gas dynamics in the divertor and in the vacuum systems represents a challenging task because of the wide range of the regimes covered by the flow. In fact, depending on the upstream plasma conditions, the Knudsen number $\mathrm{Kn}$, defined as the ratio between the molecules' mean free path and the characteristic length of the flow, spans a range from values typical of the continuum and slip flow regime above the dome, until transitional and even free molecular regime in the sub-

"See the author list of "Overview of the JET results in support to ITER" by X. Litaudon et al. to be published in Nuclear Fusion Special issue: overview and summary reports from the $26^{\text {th }}$ Fusion Energy Conference (Kyoto, Japan, 17-22 October 2016) 
divertor regions and inside the vacuum pumping ducts: to describe such a range of gas rarefaction, an approach that has been recognized to be extremely valid is the DSMC method.

DSMC is a robust and reliable tool that is able to circumvent the numerical solution of the Boltzmann equation by simulating groups of model particles whose behavior statistically mimics that of the real gas molecules and. At the same time, unlike other transport codes, it can predict the correct values of the transport coefficient (viscosity and thermal conductivity) and accurately describes non-isothermal flows. For these reasons, it is extremely suited to model the complex sub-divertor structure: studies of the neutral gas flows in both ITER [5] and JET [8] sub-divertor areas have been successfully performed with DSMC, using input data from a SOL plasma code, such as SOLPS or EDGE2D [9-10]. So far, though, the interaction between the two subsystems, namely the SOL side and the pumping, or sub-divertor, side has been one way only, with the output information in terms of particle fluxes from the edge plasma code used by the DSMC algorithm as inlet boundary conditions.

This work focuses on the DSMC simulation of the JET sub-divertor area extending it by including the feedback from the SOL in an iterative, non-automated way; the goal is to arrive at a self-consistent solution that takes into account their mutual connection in terms of neutral particle recirculation, until a consistent solution is found. Four plasma scenarios have been chosen for this purpose, and for each of them it was found that only two iterations were enough to achieve the desired result. A sensitivity analysis of the cryopump capture coefficient on the neutral particle reflux is performed as well, in order to understand how the pumping efficiency affects the flow pattern in the sub-divertor region. Results of the numerical simulations are presented in form of recirculation rates and contour plots of the quantities of practical interest.

\section{Description of the procedure}

Before trying to build any integrated package code that included both codes in an automated form, but that would have been probably unnecessary, a non-automated coupling has been applied in order to get a first estimation of the degree of interaction between DSMC and the chosen plasma code, SOLPS. In a SOLPS simulation, EIRENE applies to the pumping surfaces within the divertor geometry neutral reflection coefficients, called albedos, to mimic the effect of the neutrals being pumped outside the torus; their value is often assumed.

The purpose here is to check the validity of these assumptions using standalone, but subsequent, simulations in DSMC and SOLPS. First, an initial SOL calculation was performed, using the initial assumed albedos; the data containing the information on the neutral flux being pumped from the divertor were then used as a inlet boundary condition in a following DSMC simulation over the whole sud-divertor area: the result, in terms of neutral recirculation towards the divertor, is used to re-calculate the albedos. The next, independent SOLPS run was updated with the new values and the computed neutral flux was in turn used again by DSMC, until convergence of two subsequent albedo values was reached.

\section{Divertor Configuration}

The first main assumption that has been made is to consider a 2D model sub-divertor periodic in the z-direction (i.e. toroidal direction), in order to considerably speed up the computational time. The most important structures that have to be abandoned are the toroidal gaps between the divertor cassettes that, from ITER simulations [11] are known to have a strong influence on the overall flow and that can only be introduced in a 3D model. However, the goal here is not to model a very detailed sub-divertor structure, and future work will include the 3D modeling. A $2 \mathrm{D}$ cut of the 3D model for the JET Octant n.8 is depicted in Fig. 1 (above) and the simplified geometry used for the calculation in Fig.1 (below); previous simulations of this very same configuration [6], including also the vertical lower port and the pipes leading to the 
pressure gauge, showed that the flow field in the latter region does not have a relevant influence in the upper sub-divertor area.

Although the configuration has been simplified from the original CAD model, it does still preserve the initial high degree of geometrical complexity including the divertor coils, the radiation shielding and the cryopump as well. It is considered that only neutral particles are allowed to enter the sub-divertor domain through the two main inclined gaps located in the high field side (HFS) and low field side (LFS), and corresponding to the gaps between divertor tile 3 and 4 and between divertor tile 6 and 7, respectively. Both gaps have a similar length, namely $0.08 \mathrm{~m}$ and $0.082 \mathrm{~m}$, while the cryopump, located in the LFS, has an overall poloidal length of $0.40 \mathrm{~m}$.

\section{Boundary Conditions}

Four SOL simulations were taken from the study described in [12] for the JET vertical target configuration. Constant radial anomalous transport coefficients of $0.7 \mathrm{~m}^{2} \mathrm{~s}^{-1}$ and $0.5 \mathrm{~m}^{2} \mathrm{~s}^{-1}$ for the heat and particle diffusivities, respectively, were used for pure D simulations using the SOLPS code ([8], a combination of the multi-fluid plasma code B2 [14] [15] and the MonteCarlo neutrals code EIRENE [16] [17]). Standard sheat boundary conditions were used at the target, the specified heating was supplied at the core boundary (equally divided between the electrons and ions) and the density was controlled by a feedback loop to the requested value. The four SOLPS cases correspond to different JET plasma conditions in terms of lower and higher outer midplane separatrix electron density $\mathrm{n}_{\mathrm{e}, \text { sep }}$ and of lower and higher input power $\mathrm{P}$, namely:

Case 1) $\mathrm{P}=10 \mathrm{MW}$ and $\left\langle\mathrm{n}_{\mathrm{e}}\right\rangle_{1, \text { edge }}=2 \times 10^{19}$

Case 2) $\mathrm{P}=10 \mathrm{MW}$ and $\left\langle\mathrm{n}_{\mathrm{e}}\right\rangle_{1, \text { edge }}=4 \times 10^{19}$

Case 3) $\mathrm{P}=20 \mathrm{MW}$ and $\left\langle\mathrm{n}_{\mathrm{e}}\right\rangle_{1, \text { edge }}=2 \times 10^{19}$

Case 4) $\mathrm{P}=20 \mathrm{MW}$ and $\left\langle\mathrm{n}_{\mathrm{e}}\right\rangle_{1, \text { edge }}=4 \times 10^{19}$

The different cases were chosen with the intention to understand the impact of different plasma upstream conditions on the flow pattern and on the pumping efficiency of the subdivertor region.

The interfaces between the divertor geometry and the sub-divertor geometry are represented by the HFS and the LFS gap; the albedos associated to each gap are defined as the rate of reflected particles towards the plasma chamber, i.e. coming from the sub-divertor, versus the rate of particles pumped from the plasma chamber directed to the sub-divertor. SOLPS initally assumes the albedos to be 0.9 and 0.98 respectively for the LFS and HFS gap. 

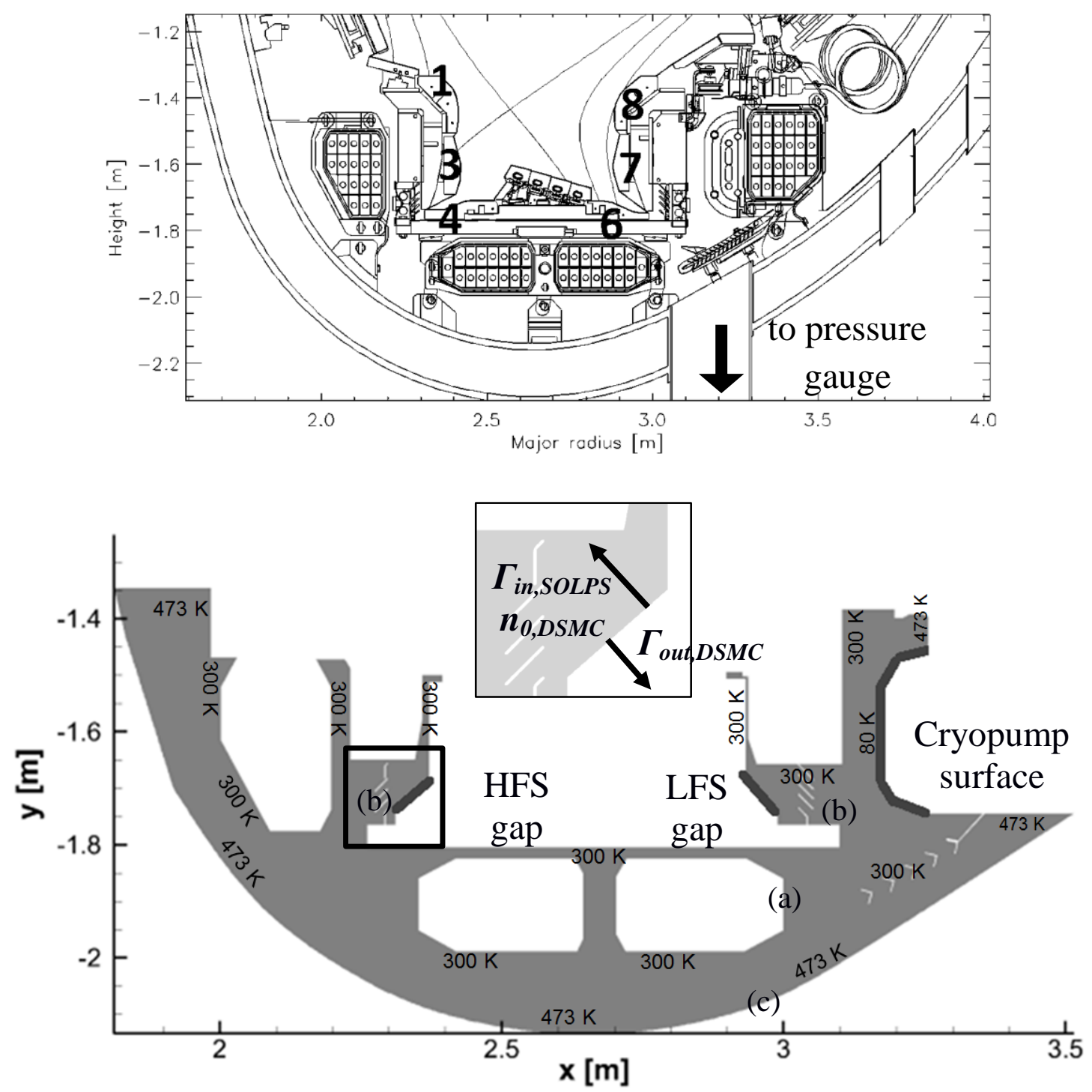

FIG. 1. 2D cut of the JET sub-divertor with divertor tiles numbering (above) and simplified geometry used in the DSMC simulation including: inlet gaps, cryopump, divertor coils (a), shielding baffles (b) and outer vessel surface (c)(below).

Both atomic and molecular deuterium in the SOL hit the divertor target and they leave the SOLPS domain: for the sake of simplicity, it has been reasonably assumed that, in close proximity of the target, a huge recombination rate for atomic neutrals into molecular neutrals occurs, so that only molecular deuterium $\mathrm{D}_{2}$ (viscosity index $\omega: 0.70$ [18]) is taken into account in the next, independent DSMC run.

In each DSMC simulation, the gas entering through the gaps is assignend with a certain number density $n_{0, D S M C}$ and a temperature $T_{0, D S M C}$. As SOLPS assumes that the far-field distribution of the gas leaving the plasma edge side to be at thermal equilibrium, the DSMC algorithm assumes the incoming gas to be at rest, i.e. the velocity distribution of each component follows a Maxwellian distribution. Based on this hypothesis, the influxes towards the sub-divertor from the gaps, output from SOLPS, can be easily converted into number density through:

$$
n_{0, D S M C}=\frac{4 \Gamma_{i n, S O L P S}}{v_{t}}
$$


Being $\Gamma_{i n, S O L P S}$ the influx from each gap as shown in Fig. 1 (right) and $v_{t}$ the thermal velocity of the far-field distribution. The DSMC input values for each plasma scenario are presented in Table 1.

TABLE 1: SUMMARY OF BOUNDARY CONDITIONS FOR DSMC CALCULATIONS.

\begin{tabular}{|c|c|c|c|}
\hline & & $n_{0}\left[m^{-3}\right]$ & $T[K]$ \\
\hline \multirow{4}{*}{ HFS gap } & $\mathrm{P}=10 \mathrm{MW},\left\langle\mathrm{n}_{\mathrm{e}}\right\rangle_{1, \text { edge }}=2 \times 10^{19} \mathrm{~m}^{-3}$ & $1.41 \mathrm{E}+19$ & 1620 \\
\hline & $\mathrm{P}=10 \mathrm{MW},\left\langle\mathrm{n}_{\mathrm{e}}\right\rangle_{1, \text { edge }}=4 \times 10^{19} \mathrm{~m}^{-3}$ & $8.07 \mathrm{E}+19$ & 1180 \\
\hline & $\mathrm{P}=20 \mathrm{MW},\left\langle\mathrm{n}_{\mathrm{e}}\right\rangle_{1, \mathrm{edge}}=2 \times 10^{19} \mathrm{~m}^{-3}$ & $7.30 \mathrm{E}+18$ & 2295 \\
\hline & $\mathrm{P}=20 \mathrm{MW},\left\langle\mathrm{n}_{\mathrm{e}}\right\rangle_{1, \text { edge }}=4 \times 10^{19} \mathrm{~m}^{-3}$ & $3.08 \mathrm{E}+19$ & 1306 \\
\hline \multirow{4}{*}{ LFS gap } & $\mathrm{P}=10 \mathrm{MW},\left\langle\mathrm{n}_{\mathrm{e}}\right\rangle_{1, \text { edge }}=2 \times 10^{19} \mathrm{~m}^{-3}$ & $9.62 \mathrm{E}+18$ & 2521 \\
\hline & $\mathrm{P}=10 \mathrm{MW},\left\langle\mathrm{n}_{\mathrm{e}}\right\rangle_{1, \mathrm{edge}}=4 \times 10^{19} \mathrm{~m}^{-3}$ & $6.00 \mathrm{E}+19$ & 1302 \\
\hline & $\mathrm{P}=20 \mathrm{MW},\left\langle\mathrm{n}_{\mathrm{e}}\right\rangle_{1, \text { edge }}=2 \times 10^{19} \mathrm{~m}^{-3}$ & $6.26 \mathrm{E}+18$ & 4364 \\
\hline & $\mathrm{P}=20 \mathrm{MW},\left\langle\mathrm{n}_{\mathrm{e}}\right\rangle_{1, \text { edge }}=4 \times 10^{19} \mathrm{~m}^{-3}$ & $1.81 \mathrm{E}+19$ & 1970 \\
\hline
\end{tabular}

The water cooled louvres and the divertor coils are assumed to be kept at a room temperature of $300 \mathrm{~K}$, while the outer wall of the vacuum vessel is assumed to be at $473 \mathrm{~K}$ [19]. Whenever a particle hits a stationary wall, then purely diffuse reflection occurs, meaning that the particle is re-emitted from the wall with a Maxwellian distribution based on the wall temperature.

The cryopump has been simulated by means of a surface kept at a temperature of $80 \mathrm{~K}$ with a given capture coefficient $\xi$, i.e. the ratio of number of particles absorbed by the pump itself versus the number of particles hitting its surface: the choice of the right value of $\xi$ is essential because of its influence on the flow field. With the assumption of gas in front of the cryopump at rest, the capture coefficient can be deduced as [20]:

$$
\xi=\frac{S}{S_{\text {id }}}=\frac{S}{A_{\text {inlet }} \sqrt{\frac{R_{0} T}{2 \pi M}}}
$$

Where $S$ is the pumping speed, $S_{i d}$ is the ideal pumping speed (i.e the speed the pump would have if all the particles hitting its surface would be absorbed), $T$ is the temperature of the pump surface, $M$ is the molar mass of the gas being pumped, $R_{0}$ is the universal gas constant and $A_{\text {inlet }}$ is the total surface of the pump. $A_{\text {inlet }}$ has been obtained multiplying the total toroidal length $(20 \mathrm{~m})$ by the length of the pump cross section calculated from the $2 \mathrm{D}$ cut in Fig.1, resulting in an area of $8 \mathrm{~m}^{2}$. Knowing that he pumping speed of the pump in situ in the JET torus is $200 \mathrm{~m}^{3} / \mathrm{s}$ [21], a capture coefficient of 0.15 has been calculated.

At the end of each DSMC simulation, the updated albedo coefficients are computed as:

$$
\alpha_{H F S / L F S}=\frac{\Gamma_{\text {out }, D S M C}}{\Gamma_{\text {in }, \text { SOLPS }}}
$$

Being $\Gamma_{\text {out }}$ the computed outflux towards the private flux region from each gap.

\section{Numerical Modelling}

The neutral flows in the sub-divertor area have been simulated using the DSMC algorithm based on the No-Time counter scheme [2]. Since DSMC has been proved a reliable method to describe the behaviour of rarefied gases, detailed descriptions have been largely covered [22], [23] and only the essential parameters of the simulations are here mentioned.

The choice of the time step is essential, because the fundamental criterion in DSMC says that it should always be a fraction of the mean collision time, in order to consistently take into account all the events affecting the motion of the particles: for all the plasma scenarios considered above, this condition is satisfied for a $\Delta t=1 \mu \mathrm{s}$. For the same reason, the grid must 
be chosen so that the cell size is a fraction of the mean free path of the molecules; in all the cases, a structured rectangular grid is used, with an average cell size of $6 \mathrm{~mm}$, resulting in an average number of cells of $2 \times 10^{4}$. Finally, the ratio of real particles versus simulated one, known as $F_{N}$, ranged from $1 \times 10^{11}$ to $1 \times 10^{12}$, resulting in a total average number of simulated particles within the simulation domain of $20 \times 10^{6}$. Using an average number of 64 cores per simulated case, the average CPU time in order to achieve full convergence is 3 to 4 days.

\section{Results and discussion}

In this section, the results of the simulations of both the plasma side and the divertor side are presented in terms of recirculation rates and contour plots. An additional sensitivity study has been performed for what concerns the sub-divertor domain, in order to investigate the factors influencing the pumping operation and, consequently, the flow patterns.

\subsection{Albedo coefficients for HFS and LFS}

Two rounds of iterations between SOLPS and DSMC were necessary to reach converged values of the albedos of the two communication gaps between SOL and sub-divertor, i.e the procedure was stopped because an error of less than 3\% was found on two subsequent calculated albedos. Fig. 2 shows the convergence of the iterations towards the final results, which are presented in Table 1. In all the four considered plasma cases, the final recirculation rates show a discrepancy of $\sim 5 \%$ with respect to the original ones assumed for the LFS gap, and of $\sim 9 \%$ to the ones assumed for the HFS gap: while the original SOLPS calculations have assumed higher recirculation fluxes from the sub-divertor towards the edge plasma, the new coupled runs proved that the actual pumping efficiency is, although slightly, better, i.e. the outfluxes towards the edge plasma are lower. Reasonably, the DSMC simulations resulted as well in values of the coefficients related to the LFS gaps that are lower than those for the HFS, due to the intrinsic asymmetrical position of the cryopump, directly facing the LFS gap. Since each gap presents the same trend in terms of albedos for all four considered cases, the updated values that could be considered in future SOLPS simulations are:

- $\alpha_{\mathrm{HFS}}=0.90($ previous one $=0.98)$

- $\alpha_{\text {LFS }}=0.85$ (previous one $\left.=0.90\right)$. 
TABLE II: UPDATED VALUES FOR ALBEDO COEFFICIENTS FOR HFS AND LFS GAPS.

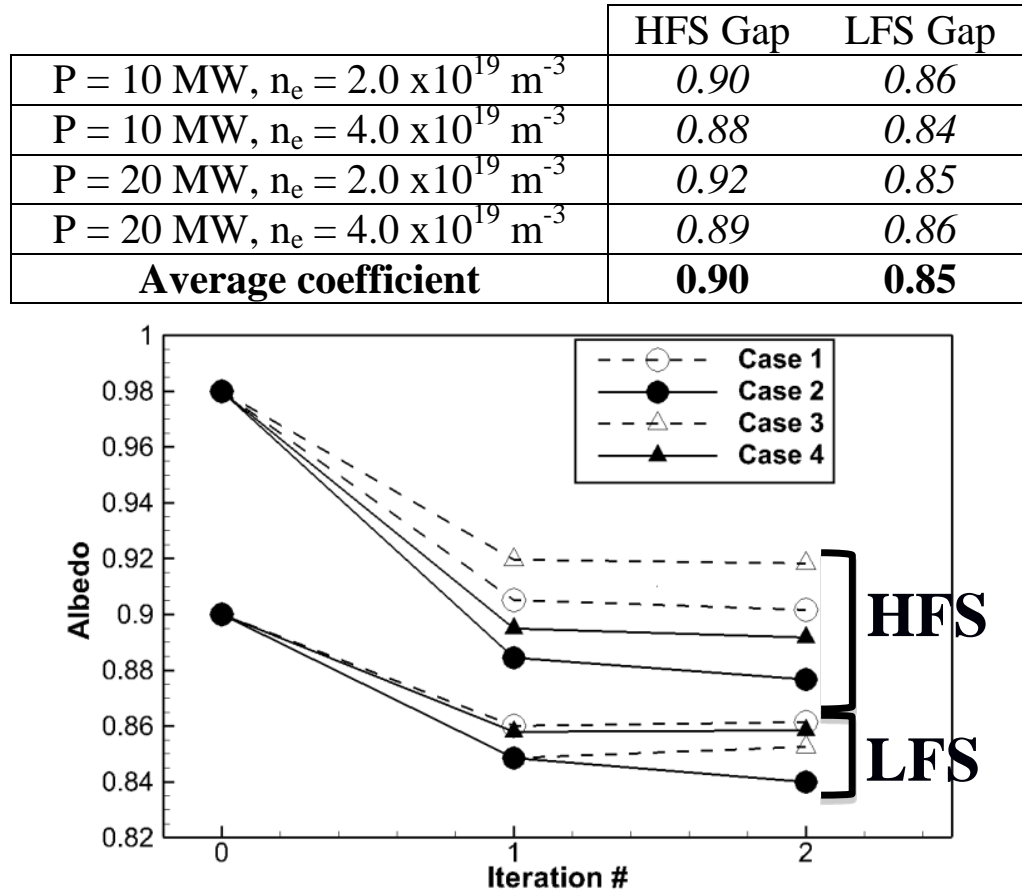

FIG. 2: Convergence of the albedo coefficients for the different considered plasma scenarios.

Contour plots of the two most representative cases are compared in Fig. 3. Results showed that the plasma parameter having the higher influence on the flow patterns is the electron density: in the low and high density case, the Kn number spans from 0.2 to 4 , still remaining in the transitional, collisional flow range which is well described by DSMC. The Kn number has been calculated in post-processing as the ratio between the mean free path and a length characteristic of the system, which in this case has been chosen to be an average between the two inlet gap lengths, namely $0.81 \mathrm{~m}$. The $\mathrm{D}_{2}$ density contour plots also clearly explains the high recirculation rates that occur through the gaps: the incoming fluxes from the divertor hit the shielding louvres and remain confined in close proximity of the inlet, creating regions where the particle density is higher than in the rest of the structure and the gas particles themselves are reflected by the louvres towards the main chamber again. In addition, the temperature contours (Fig.3 - bottom right) definitely highlights the strong non-isothermal flow pattern, whose complexity the DSMC method is well suited to represent.

\subsection{Pumping efficiency studies}

The impact on the pumping performance of different pumping and geometrical configurations in the sub-divertor area has been studied.

- For the plasma case $1\left(\mathrm{P}=10 \mathrm{MW},\left\langle\mathrm{n}_{\mathrm{e}}\right\rangle_{1, \text { edge }}=2 \times 10^{19} \mathrm{~m}^{-3}\right)$, a parametric study of the capture coefficient has been performed in order to assess the pumping efficiency of the cryopump in situ of the torus. Three additional, increasing values of $\xi$ have been chosen $(0.3,0.6,1.0)$ and the corresponding pumped particle flux has been computed; the latter has then been normalized with the total incoming particle flux, which is the sum of the influxes from both gaps, equal to $9.77 \times 10^{21} \mathrm{~s}^{-1}$. Fig.4 shows the results of the DSMC simulation for $\xi=1.0$ : the number density, with respect to Fig.3 (top), is obviously decreasing towards the pumping side due to the increased ability of the pump to capture more particles. Nevertheless, on a closer look in Fig.5 (left), it can be seen that, even in the case of an ideal pump absorbing every particle hitting its surface, there is no relevant improvement in efficiency; in the best case scenario, only $16 \%$ of the sub-divertor 
incoming exhaust flux would be removed. The low sensitivity of the system to a dramatic increase in $\xi$ is due to the combined effect of the intrinsic asymmetrical position of the cryopump, located on the LFS, and the presence of the divertor coils and water-cooled baffles, which significantly limit the conductance of the overall structure.

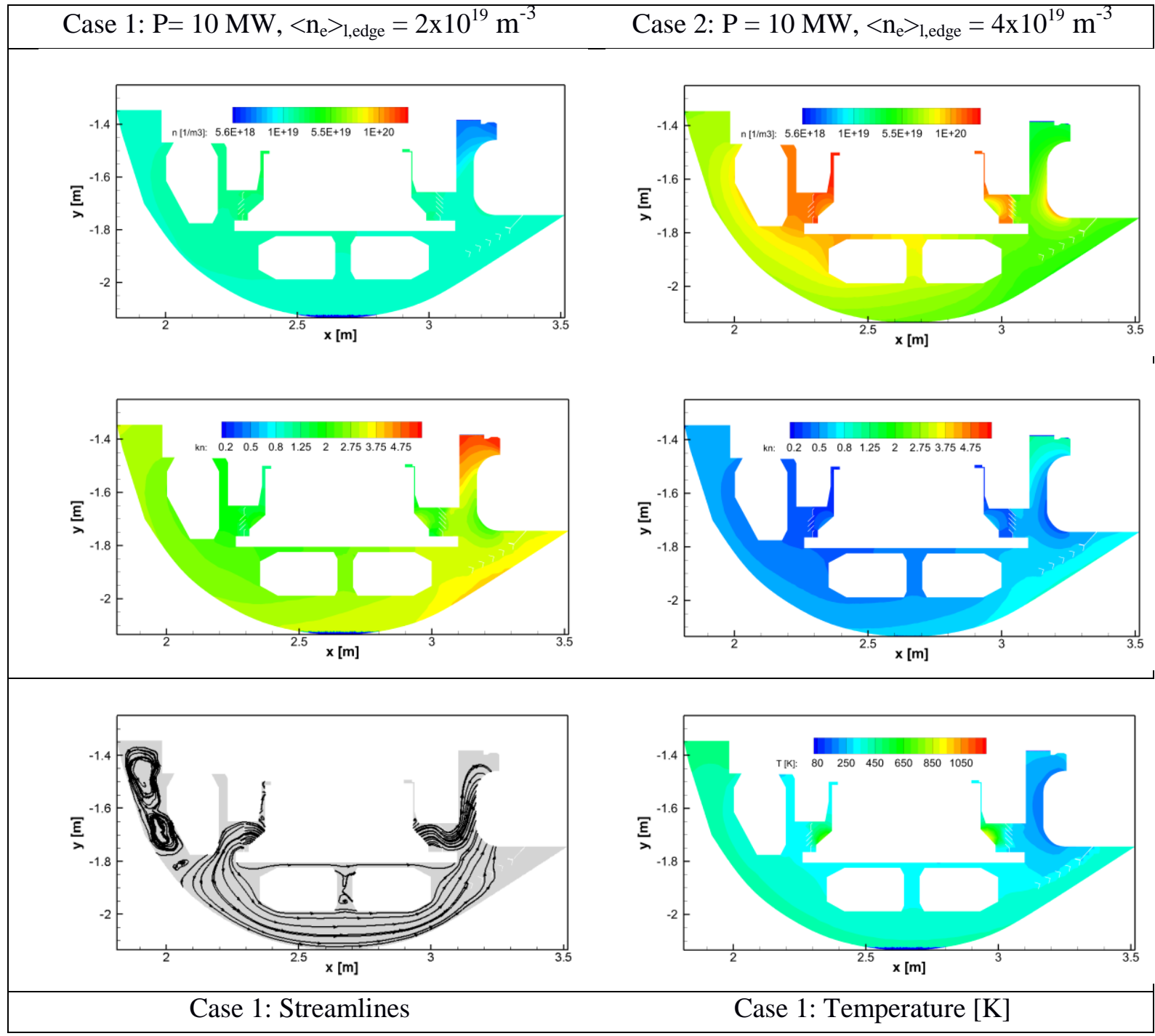

FIG. 3: $D_{2}$ number density (top) and Kn number (middle) contours from DSMC calculations for Case 1 and Case 2. Bottom: Streamlines towards the cryopump (left) and temperature contour plot (right) for Case 1.

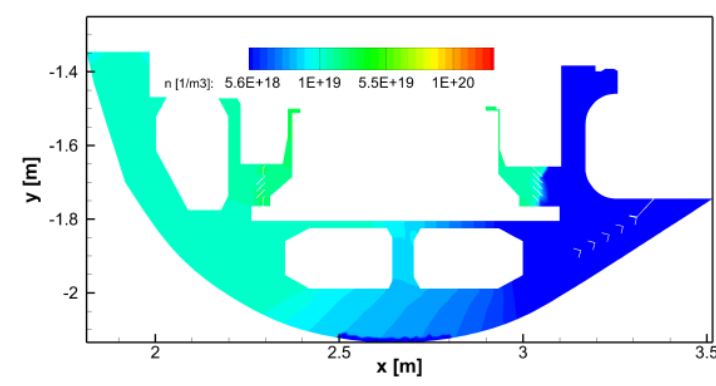

FIG. 4: $D_{2}$ number density for the case $\xi=1.0$. 

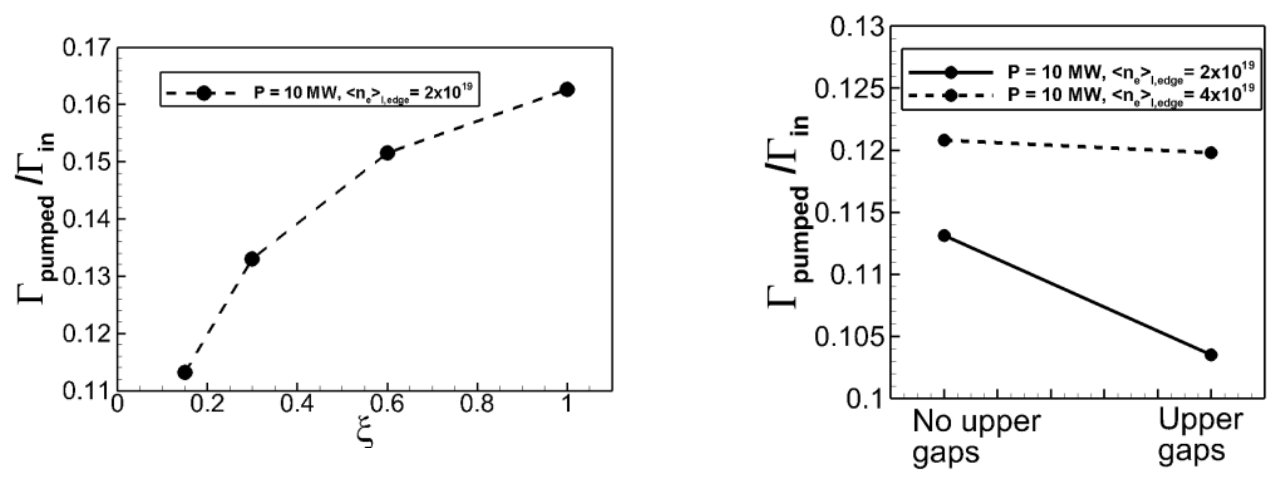

FIG. 5: Normalized pumped particle flux versus capture coefficient $\xi$ (left) and in the case of open upper gaps (right).

- In order to present the influence of the geometrical inlet configuration on the entity of the recirculation rates towards the main chamber, two additional upper gaps, namely the upper gaps between tile 1 and 3 and between tile 7 and 8 (see Fig. 1 - left), have been considered as open gaps. In the simulation for Case 1 and $2, \mathrm{D}_{2}$ particles are considered to be canceled from the DSMC domain, representing their recirculation and following re-ionization in the plasma chamber. Like in the previous study, as presented in Fig. 5 (right), in both plasma cases taken into account, the impact of the updated flow configuration does not significantly affect the flow field and the pumping efficiency: variation with respect to the nominal cases is within the $1 \%$ of the overall influxes. The reason of this result could be found in the geometrical feature of the gaps/outlets, whose smaller openings allow only a small fraction of the gas to escape, while the main flow pattern is still driven by the pump itself and by the main gaps.

\section{Summary}

The present work includes an integrated analysis of neutral deuterium gas dynamics in the JET divertor and sub-divertor. Two well-known approaches, the SOLPS code for the plasma side and the DSMC algorithm for the sub-divertor side have been coupled until convergence was reached in terms of neutral recirculation fluxes.

Four JET divertor scenarios are studied, two with higher and two with lower electron density, each considering two different values of the input power, and the calculations of the $\mathrm{D}_{2}$ flow fields are shown. The pressure driven flows established in the divertor structure generate anyways a high amount of particle exhaust flowing back towards the main chamber, but with a rate that was found smaller of 5\% to $9 \%$ with respect to the assumed values in SOLPS original simulations. The small relaxation that could then be introduced in the assumption of the albedos is, obviously, strictly valid only for the specific JET configuration taken into account; further verifications could be carried out for other divertor arrangements (e.g. vertical target) but the so far obtained results seems promising for they also show little discrepancies with the assumed values in other modeling activities performed with SOLPS or EDGE2D-EIRENE [24] [25].

Further analysis on the sub-divertor geometry showed that the influence of the upper gaps on the recirculation and on the pumping flows is irrelevant and, therefore, they could be completely neglected on any future work on this configuration.

In the future, the DSMC algorithm could be further used to include gas mixtures (e.g. mixtures of $\mathrm{He}, \mathrm{D}$ and $\mathrm{D}_{2}$ ) and extended for $3 \mathrm{D}$ modeling, required to investigate the toroidal flow effects. 


\section{Acknowledgments}

This work has been carried out within the framework of the EUROfusion Consortium and has received funding from the Euratom research and training programme 2014-2018 under grant agreement No 633053. The views and the opinions expressed herein do not necessarily reflect those of the European Commission.

The computational resources needed for this work were provided by the Helios Supercomputer at IFERC-CSC.

\section{References}

[1] KUKUSHKIN, A., et al., "Effect on neutral transport on ITER divertor performance", Nucl. Fusion 45 (2005) 608.

[2] BIRD, G. A., Molecular Gas Dynamics and the Direct Simulation of Gas Flows, Oxford University Press, Oxford (1994).

[3] LOARTE, A., "Effects of divertor geometry on tokamak plasmas", Plasma Phys. Control. Fusion 45 (2001) 183-224.

[4] STOTLER, D. P., "Three-dimensional simulation of gas conductance measurement experiments on Alcator C-Mod”, J. Nucl. Mater. 337 (2005), 510-514.

[5] GLEASON-GONZÁLEZ, C., et al., "Simulation of neutral gas flow in a tokamak divertor using the DSMC Method”, Fus. Eng. Des. 89 (2014), 1042.

[6] MOULTON, D., et al., "Pumping in vertical and horizontal target configurations on JET in L-mode; an interpretative study using EDGE2D-EIRENE", (Proc. $42^{\text {nd }}$ Conference on Plasma Physics, Lisbon, 2015), EPS (2015), Vol. 39E.

[7] KUKUSHKIN, A., et al., "Finalizing the ITER divertor design: the key role of SOLPS modeling”, Fus. Eng. Des. 86 (2011) 2865-2873.

[8] VAROUTIS, S., et al., "Simulaton of Neutral Gas Flow in the JET subdivertor", submitted to Fus. Eng. Des. (2016).

[9] REITER, D., "Progress in two-dimensional plasma edge modelling", J. Nucl. Mater. 196-198 (1992), 80.

[10] WIESEN, S., "EDGE2D-EIRENE code interface Report", JET ITC-Report (2006), http://www.eirene.de/e2deir_report_30jun06.pdf.

[11] HAUER, V., et al., "Conductance Modelling of ITER vacuum systems", Fus. Eng. Des. 84 (2009), 903-907.

[12] COSTER, D., "Detachment physics in SOLPS simulations", J. Nucl. Mater. 415 (2011), S545.

[13] SCHNEIDER, R., et al., "Plasma edge physics with B2-Eirene", Contrib. to Plasma Phys. 45 (2006), 3.

[14] BRAAMS, B. J., Computational studies in Tokamak Equilibrium and Transport, PhD Thesis, Univ. of Utrecht, Netherlands (1986).

[15] BRAAMS, B. J., "Radiative Divertor Modelling for ITER and TPX", Contrib. to Plasma Phys. 36 (1996), 276. 
[16] REITER, D., "Progress in two-dimensional plasma edge modelling", J. Nucl. Mater. 196-198 (1992), 80.

[17] REITER, D., et al., "Time dependent neutral gas transport in tokamak edge plasma”, J. Nucl. Mater. 220-222 (1995), 987.

[18] CHAPMAN, S., COWLING, T.G., The Mathematical Theory of Non-uniform gases, University Press, Cambridge (1952).

[19] KRUEZI, U., Private communication, 2014.

[20] HAEFER, R.A., Cryopumping, Clarendon Press, Oxford (1989).

[21] OBERT, U., et al., "Performance of the JET pumped divertor cryopump system", (Proc $16^{\text {th }}$ IEEE/NPSS Symposium on Fusion Eng. Illinois, 1995), IEEE (1995), 742-745.

[22] GALLIS, M., et al., "Direct Simulation Monte Carlo: the quest for speed" (Proc. AIP Conf), vol. 1628, 27 (2014).

[23] SCHEN, C., Rarefied Gas Dynamics, Springer, Berlin, Heidelberg (2005).

[24] GUILLEMAUT, C., "Influence of atomic physics on EDGE2D-EIRENE simulation of JET divertor detachment with carbon and beryllium/tungsten plasma-facing components", Nucl. Fusion 54 (2014), 093012.

[25] BELO, P., "Modelling the carbon distribution in JET L-Mode plasmas in A MKIIHD divertor with EDGE2D/EIRENE", EFDA-JET-CP05/32 Report (2010). 